\title{
Automatic Apparatus for Simultaneous Measurement of Resistance and Inductance Temperature Transition Dependences of Superconductors
}

\author{
A. Koňakovský, A. Cigáň, J. Maňka, J. Bartkovjak \\ Institute of Measurement Science, Slovak Academy of Sciences, Dúbravská cesta 9, 84104 Bratislava, Slovakia, \\ anton.konakovsky@savba.sk
}

\begin{abstract}
An automatic measurement complex for the study of transition properties of HTc superconductors using the transport method of the measurement of the resistance temperature dependence and the contactless mutual inductance method of the measurement of temperature dependence of the induced voltage based on the Meissner-Ochsenfeld effect have been developed. In addition, temperature dependences of harmonic components of the induced voltage may be measured simultaneously using the Brüel \& Kjaer signal analyzer. Results of transport and induced voltage measurements are compared using YBCO superconductors with distinct grain and intergrain properties prepared by different technological procedures. The question of the mutual compatibility of such methods is discussed.
\end{abstract}

Keywords: high-temperature superconductors, transition characteristics, resistance method, mutual inductance method, harmonic response, critical temperature

\section{INTRODUCTION}

卫 BETTER UNDERSTAND properties of hightemperature superconductors, it is often necessary to separate, within characteristics measured, intergrain and intragrain contributions. This is especially important for basic characteristics of superconductors as well, such as the critical temperature, critical current density, or critical magnetic field values. It is even more significant for their heavy-current applications.

The critical temperature $T_{\mathrm{c}}$ is one of the basic characteristics of the superconducting state. It is determined, as a standard, by means of transport measurement of dependence of electric resistance $R$ or resistivity on temperature $T$ during the transition of sample from the normal to the superconducting state. However, various other methods are used, e.g. inductance and magnetic, but the question of their compatibility is still open. The four-point measurement technique of the $R$ vs. $T$ dependence is the best-known standard method for the determination of various characteristics of superconducting and normal states of superconductors. The critical temperature $T_{\mathrm{c}}(R=0)$ and the transition width $\Delta T_{\mathrm{c}}(R=0)$, characterized by $10-90 \%$ criteria, are the best-known from these characteristics. Other two contactless measuring methods - using a change of the selfinductance of the coil located in the vicinity of the measured sample or using a change of the mutual inductance of two coils separated by a sample - are also used. For the given two inductance methods of transition characteristic measurements, in the sense of the characteristic critical temperature $T_{\mathrm{c}}(R=0)$, the $T_{\mathrm{c}}{ }^{\text {on }}$ determined from the onset of a diamagnetic behaviour (decrease in the induced voltage) is usually used. However, in case of the transition width $\Delta T_{\mathrm{c}}(R=0)$, determined from electric resistance measurements, and $\Delta T_{\mathrm{c}}^{\text {ind }}$ obtained from temperature dependence of induced voltage, there is no consensus, except that the higher $\Delta T_{\mathrm{c}}$ is, the worse superconducting properties are in both cases. In addition, the transition width is an important gauge of homogeneity of the prepared samples. There are several papers, in which characteristics of inductance and resistance measurements are mutually compared, but not in a single transition temperature cycle as known by us.

This paper presents a synchronized scanning of the transition process by the resistance and inductance methods. For this purpose, an automatic laboratory apparatus $[1,2]$ has been modified and tested by means of $\mathrm{YBa}_{2} \mathrm{Cu}_{3} \mathrm{O}_{7-\delta}$ superconductor samples, prepared for this purpose by special technological procedures. Results of the harmonic response of induced voltage signal within the superconductive transition interval of samples are also shown and commented.

\section{APPARATUS FOR MEASUREMENT OF TEMPERATURE TRANSITION CHARACTERISTICS}

\subsection{CRYOGENIC PROBE}

Cryogenic probe comprises two multi-turn primary and secondary coils of the diameter of 10 and $5 \mathrm{~mm}$ and inductance of $L=11.5 \mathrm{mH}$ and $0.25 \mathrm{mH}$, respectively, made from $\mathrm{Cu}$ wire. The measured samples of superconductors, in the form of pellets, with the diameter of about $12 \mathrm{~mm}$ and the thickness of 1 to $3 \mathrm{~mm}$ are inserted between the two coils. The primary coil is supplied by sinusoidal current. The Pt100 sensor, in the form of a planar bifilarly wound coil with the diameter of $12 \mathrm{~mm}$, is attached to one of the sample faces in order to obtain a good thermal contact with the sample. All the parts are encapsulated in a thermally insulated vessel to reduce velocity of temperature changes for a reliable determination of the temperature values corresponding to the data measured. Amplitude of the harmonics induced voltage ( $y$-coordinate of one data point, e.g. in Fig.3, down) was obtained as mean from eight measurements of voltage spectra. Linear approximation was used for a determination of corresponding temperature values. The electric contacts of the samples have been prepared from an indium solder. 


\subsection{MEASURING APPARATUS}

The measuring apparatus consists of a Dewar vessel, electronic devices, cryogenic probe with a sample and a precise digital voltmeter connected to a $\mathrm{PC}$ and electronic switch. The block diagram of the electronic apparatus is shown in Fig.1.

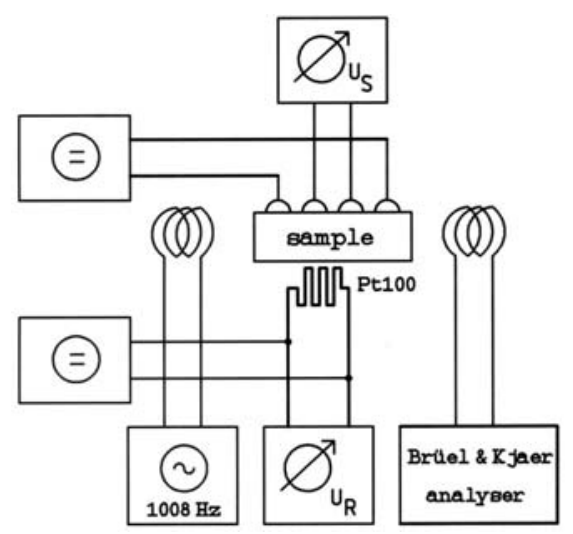

Fig.1 Simplified block diagram of the electronic apparatus used for measurement of transition characteristics of superconductors.

The apparatus comprises a precision AC current source for the primary coil, the Pt100 temperature sensor, two current sources for temperature sensor and for the measurement of the resistance of sample, a voltmeter for the measurement of the temperature and the resistance of the sample and the Brüel \& Kjaer signal analyzer for the measurement of the amplitude of frequency harmonics of the transition induced voltage.

\subsection{THE ELECTRONIC SWITCH AND SOFTWARE FOR PRECISION MEASUREMENT OF TEMPERATURE AND ELECTRIC RESISTANCE}

Values of observed characteristics were measured by Solatron digital voltmeter No. 7081 using the electronic switch. Voltmeter terminals are gradually interconnected by software and electronic switch with terminals of the Pt100 temperature sensor, terminals of the $\mathrm{RN}$ resistance standard (which serves for the measurement of DC current of the temperature sensor) and with terminals of the sample contacts and secondary detection coil, respectively. The defined magnitude of temperature change is used for the controlling the start the measured data cycles.

\subsection{MEASURED SAMPLES}

Samples of the $\mathrm{YBa}_{2} \mathrm{Cu}_{3} \mathrm{O}_{7-\delta}$ superconductors with a different microstructure have been prepared. The S1-sample of the $\mathrm{YBa}_{2} \mathrm{Cu}_{3} \mathrm{O}_{7-\delta}$ composition is a high-grade polycrystalline one, which has been prepared by the solid-state reaction method, however, technological parameters have been set up in such a way that the volume superconducting properties, e.g., the volume magnetization, have been maximized. The S2-sample of the same composition has been prepared by a standard quench melt growth process. The S2 sample shows no intergrain weak links. This has been verified by optical polarized microscopy and detailed magnetization measurements.

\section{RESULTS AND DISCUSSION}

Typical dependences of the electrical resistance and the RMS values of induced voltage in the secondary coil at driving frequency of $1008 \mathrm{~Hz}, \mathrm{U}^{*}$, with a subtracted effect of background (a sample-free probe), are shown in Fig.2.
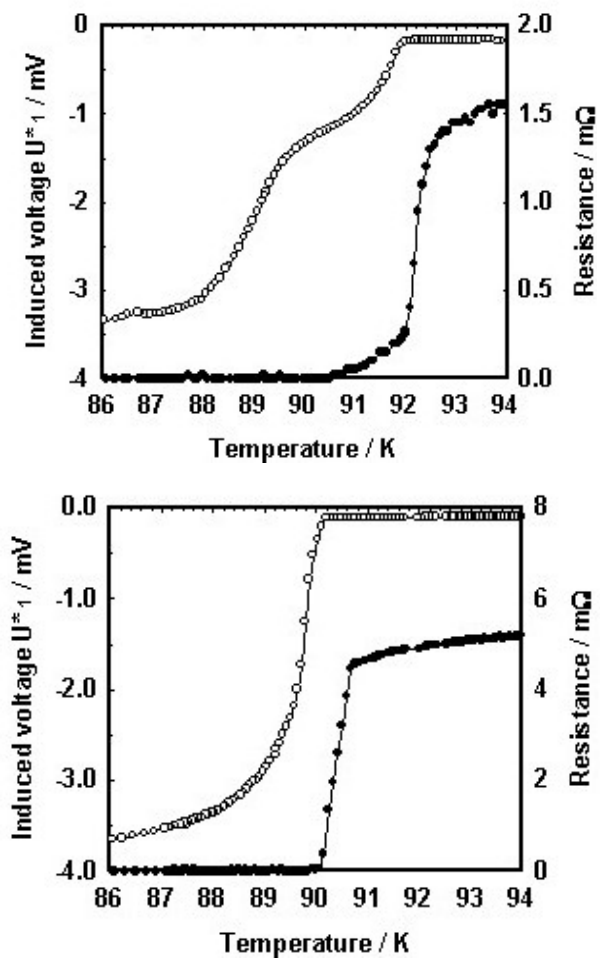

Fig.2 Temperature dependences of the resistance $(\bullet)$ and the induced voltage (O) at the frequency of $1008 \mathrm{~Hz}$ of the polycrystalline $\mathrm{YBa}_{2} \mathrm{Cu}_{3} \mathrm{O}_{7-\delta}$ sample (up) and the textured fewdomain $\mathrm{YBa}_{2} \mathrm{Cu}_{3} \mathrm{O}_{7-\delta}$ sample (down) in the area of transition into the superconducting state.

The following values of resistance $\left(T_{\mathrm{c}}, \Delta T_{\mathrm{c}}\right)$ and inductance $\left(T_{\mathrm{c}}{ }^{\text {on }}\right)$ characteristics have been determined for the polycrystalline S1 sample: $90.5 \mathrm{~K}, 1.0 \mathrm{~K}$ and $91.9 \mathrm{~K}$, respectively; and for the few-domain $\mathrm{S} 2$ sample, the following ones have been determined: $90.1 \mathrm{~K}, 0.45 \mathrm{~K}$ and $90.7 \mathrm{~K}$. Contrary to the transport $R(T)$ behaviour, the $U^{*}{ }_{1}(T)$ dependence of the S-sample shows a step-like behaviour in the superconducting transition interval. The different behaviour of the two samples is also illustrated by amplitude of their odd harmonic signals, Fig.3. In addition, non-zero even harmonic components of induced voltages could have also been observed, which could indicate the presence of a DC component of the magnetic field of a currently unknown origin.

It can be deduced from the temperature dependences of the individual harmonics voltages that the generation of the harmonic components occurs mainly in the area of a transition into the superconducting state. In general, the effect of higher harmonics generation can be ascribed to the existence of nonlinear effects. Their reasons are still being examined. Thermal oscillation from power dissipation or 
depairing effects have been considered as such reasons, [3]. In addition, high- $T_{\mathrm{c}}$ superconductors are materials, for which nonlinearities are intrinsic. They are related to the existence of the intragrain (connected with the layered structure of HTc superconductors) or intergrain structural inhomogeneities, showing properties of Josephson junctions (JJs). The JJs are characterized by the Josephson`s nonlinear electric current magnetic flux relation.
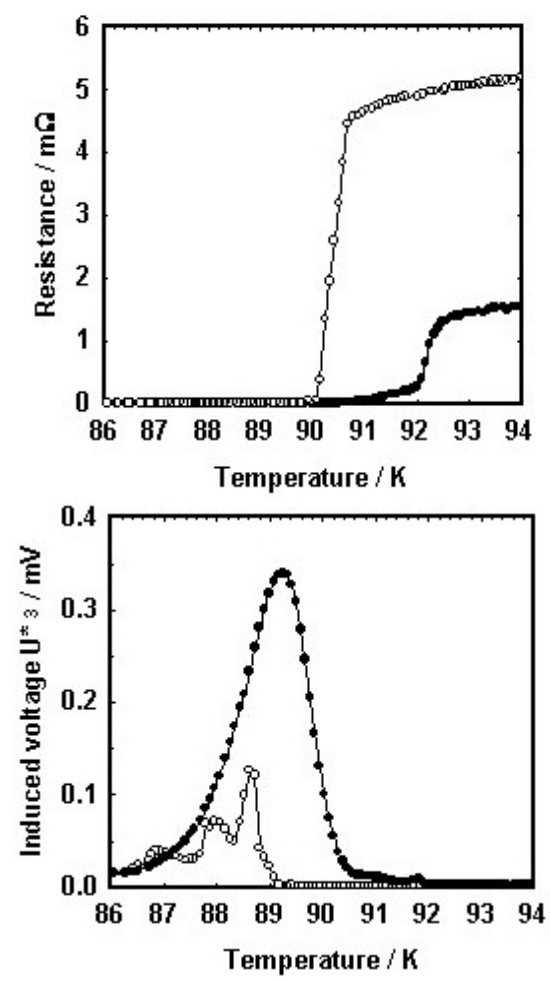

Fig.3 Temperature dependences of the resistance (up) and the $3^{\text {rd }}$ harmonic of the induced voltage $U_{3}^{*}$ (down) of the polycrystalline S1 $(\bullet)$ and the textured few-domain $\mathrm{S} 2(\circ)$ samples of $\mathrm{YBa}_{2} \mathrm{Cu}_{3} \mathrm{O}_{7-\delta}$ in the area of a transition into the superconducting state.

In polycrystalline samples, there are intergrain JJs that limit the transport critical current density and its dependence on magnetic field. It is known that the single crystal or melttextured, mono- or few-domain samples, like S2, do not show intergrain weak links, however, inside the grains, there are also inherent inter- and intra- unit- cell JJs, [4]. There are also other explanations for the non-linear response of HTc superconductors, [5]. Regardless of this, measurements of the $3^{\text {rd }}$ harmonic of the induced voltage are usually used to estimate the critical current density in superconducting structures. The relationship between the amplitude of the $3^{\text {rd }}$ harmonics of the induced voltage and the critical current density has been investigated in several publications, [6-8].

Comparing resistance and induced voltage transition characteristics, it can by inferred that they are partially complementary. So, resistance characteristics include rather surface properties (intergrain) and inductance characteristics and their harmonic components include more information about volume (inhomogeneity) properties.

\section{CONCLUSIONS}

We presented an automatic improved apparatus allowing measurements of transition transport and inductance characteristics and corresponding harmonic signals in a single course. It can be a very useful tool for analyzing the high temperature superconductors as well as for a deeper understanding of limits of transport and inductance methods of measuring the transition curves and adequate interpretation of the data measured.

\section{ACKNOWLEDGEMENTS}

This work was supported by the Slovak Grant Agency for Science, Project No. (2/7083/27), and by the Slovak Research and Development Agency.

\section{REFERENCES}

[1] Koňakovský, A., Cigán̆, A., Maňka, J., Buchta, Š. (2002). Contactless measurement of transition characteristics of HTc superconductors. Journal of Electrical Engineering, 53 (10/S), 164-166.

[2] Koňakovský, A., Cigáň, A., Maňka, J., Buchta, Š. (2003). Simultaneous measurement of the resistance and inductance transition characteristics of the HTc superconductors. Measurement Science Review, 3, 115117.

[3] Ossandón, J.G., Giordano, J.L., Esquinazi, P., Schaufuss, U., Sergeenkov, S. (2006). Non-linear response of ac conductivity in narrow YBCO film strips at the superconducting transition. Journal of Physics: Conference Series, 43, 655-658.

[4] Ling, D.C., Yong, G., Chen, J.T., Wenger, L.E. (1995). Experimental evidence for intra- and inter-unit-cell Josephson junctions in a $\mathrm{YBa}_{2} \mathrm{Cu}_{3} \mathrm{O}_{7-\delta}$ single crystal. Physical Review Letters, 75, 2011-2014.

[5] Ji, L., Sohn, R.H., Spalding, G.C., Lobb, C.J., Tinkham, M. (1989). Critical-state model for harmonic generation in high temperature superconductors. Physical Review B, 40, 10936-10945.

[6] Mawatari, Y., Yamasaki, H., Nakagawa, Y. (2003). Inductive measurements of third-harmonic voltage and critical current density in bulk superconductors. Applied Physics Letters, 83, 3972-3974.

[7] Kamitani, A., Ikuno, S., Takayama, T. (2005). Transient response of shielding current density in HTS. Physica C, 426-431, 804-808.

[8] Nadami, T., Otabe, E.S., Kiuchi, M., Matsushita, T., Mawatari, Y., Yamasaki, H., Nakagawa, Y. (2005). Numerical calculation of third harmonic voltage induced by a shielding current in a superconducting thick film. Physica C, 426-431, 688-692. 